Check for updates

Cite this: RSC Adv., 2017, 7, 23866

\section{Au@Ag core-shell nanoparticles with a hidden internal reference promoted quantitative solid phase microextraction-surface enhanced Raman spectroscopy detection $\dagger$}

\author{
Lei Sun, Min Zhang, Vinothkumar Natarajan, Xiaofei Yu, Xiaoli Zhang* \\ and Jinhua Zhan (ID) *
}

\begin{abstract}
The combined applications of solid phase microextraction coupled with surface enhanced Raman spectroscopy (SPME-SERS) detection have been successfully used in environmental analysis. In this work, ZnO nanorods (ZnO NRs) decorated with Au(14-ATP@Ag core-shell nanoparticles (NPs) were fabricated on a stainless steel fiber. The inclusion of an internal reference significantly improved the quality of the quantitative SERS measurements of the analytes. The obtained SERS-active SPME fiber resulted in a better reproducibility of the SERS signals. The relative standard deviation (RSD) value of the SERS-active SPME fiber was found to be $6.7 \%$ using malachite green (MG) as a probe molecule. A RSD value of $6.8 \%$ was observed when the SERS-active SPME fiber was exposed to laser irradiation continuously for about $10 \mathrm{~min}$. These results suggest that the fiber was prepared with good stability and uniformity. Quantitative SERS measurements of the SERS-active SPME fiber showed an excellent quantitative ability for the detection of $M G$ and crystal violet $(C V)$ in aquaculture water. In $\mathrm{CV}$, the results showed a good linear relationship in the concentration range of $5 \times 10^{-8} \mathrm{M}$ to $5 \times 10^{-6} \mathrm{M}$, and the limit of detection (LOD) can reach $1.9 \times 10^{-9} \mathrm{M}(\mathrm{S} / \mathrm{N}=3)$. This result indicates that the inclusion of an internal reference can effectively correct the signal fluctuation. This work provides versatile detection capability for rapid on-site quantitative SERS detection of different molecular species in environmental media.
\end{abstract}

Received 17th March 2017 Accepted 24th April 2017 DOI: $10.1039 / \mathrm{c} 7 \mathrm{ra03164e}$ rsc.li/rsc-advances
Conventional sampling methods require large amounts of solvents, long extraction times, and multiple steps. Therefore, a new sample preparation technique with simple, sensitive and time-efficient has becomes a hotspot issue of current research. Prior to analysis, volatile and semivolatile substances in a matrix or in solution may be preconcentrated without more laborious manipulations. The following techniques such as liquid extraction (LE), solid-phase extraction (SPE) and solid phase microextraction (SPME) are powerful pre-concentration procedures for extraction of pollutant in environmental monitoring. Particularly, SPME is a simple, solvent-free on-site sampling method for separating and preconcentrating the target compounds of low concentration from various sample matrices. ${ }^{4-6}$

Many studies have reported that SPME coupled with gas chromatography (GC), 7 gas chromatography-mass spectrometry (GC-MS), ${ }^{8}$ high performance liquid chromatography (HPLC) and capillary electrophoresis (CE) for the detection of analytes in biological, environmental and food analysis. ${ }^{9,10}$ Further, various spectroscopic methods are also coupled with SPME in the detection of analytes. Daniel et al. investigated SPME coupled infrared spectroscopy technique for the detection of a variety of organic compounds such as benzene, chlorobenzene, toluene, chloroform, and $p$-chlorotoluene in water. The authors showed that SPME-IR could be used to detect the individual compounds in
Key Laboratory for Colloid \& Interface Chemistry of Education Ministry, Department of Chemistry, Shandong University, Jinan, Shandong, 250100, P. R. China. E-mail: jhzhan@sdu.edu.cn

$\dagger$ Electronic supplementary information (ESI) available. See DOI: 10.1039/c7ra03164e 
mixtures by extracting aromatic compounds from the gasoline contaminated water samples. ${ }^{11,12}$

Raman spectroscopy is a powerful tool to acquire information about the vibrational modes of molecules. An inelastically scattered light by the molecular vibrations of a given molecular structure is collected to form a Raman spectrum. ${ }^{13}$ Raman spectroscopy is also a powerful tool in environmental monitoring. ${ }^{14-17}$ Brian and David demonstrated that BTEX could be detected in 2-3 orders of magnitude using SPME coupled Raman spectroscopy. Their detection limits were in the range of 1-4 ppm. The authors also observed the excellent selectivity for the detection of BTEX compounds by SPME..$^{14,18}$ However, the low strength of Raman signals is the main drawback associated with this technique.

SERS technique can be used to greatly amplify the Raman signals of the molecules and has been considered to be an ultrasensitive label-free detection method, which greatly extends the application of Raman spectroscopy. ${ }^{19-21}$ SERS technique has been applied to a variety of applications..$^{22-28}$ There have been efforts to combine the advantages of SPME and SERS for rapid detection of environmental pollutants. ${ }^{29-31}$ The critical issues associated with the application of SERS as a routinetechnique is the variation of enhancement factors from one substrate to another and also from different regions of the same substrate. $^{32,33}$ Considering the challenges encountered, many research efforts have been focused to achieve efficient quantitative SERS measurements using SERS substrates. ${ }^{34}$

Herein, we reported the SERS-active SPME fiber using Au@4ATP@Ag NPs for the quantitative detection of analytes in water. 4-ATP simultaneously served as the internal SERS reference. A simple and on-site SPME-SERS method was proposed for the detection of pollutants in aquaculture water. The quantitative measurements of two analytes, MG and CV, are shown in this study to demonstrate the detection capability of this scheme. This work highlights the potential application of this technique for rapid on-site analysis of environmental pollutants.

\section{Experimental section}

\subsection{Chemicals and reagents}

Stainless steel fibers (O.D., $0.20 \mathrm{~mm}$ ) were purchased from the An Ting Micro-Injector Factory (Shanghai, China). Zinc nitrate hexahydrate $\left(\mathrm{Zn}\left(\mathrm{NO}_{3}\right)_{2} \cdot 6 \mathrm{H}_{2} \mathrm{O}\right)$ and hexamethylenetetramine (HMT) were provided by Sinopharm Chemical Reagent Co. Ltd. (Shanghai, China). 4-Aminothiophenol (4-ATP, 97\%) was obtained commercially from Sigma-Aldrich. Silver nitrate $\left(\mathrm{AgNO}_{3}\right)$, sodium citrate, chloroauric acid $\left(\mathrm{HAuCl}_{4}\right), 3$-aminopropyltriethoxysilane (APTES), dimethyl sulphoxide (DMSO), malachite green, crystal violet, acetone and ethanol were procured from Sinopharm Chemical Reagent Co., Ltd. (Shanghai, China). Ultrapure water $(18.25 \mathrm{M} \Omega \mathrm{cm})$ was used in the experiments. All reagents used in the experiments were analytical grade and used as received without further purification.

\subsection{Characterization techniques}

The morphology of the SERS-active fiber was characterized by using a scanning electron microscope (JSM-6700 field-emission scanning electron microscope). Transmission electron microscopy (TEM) images were obtained using a JEOL JEM-1011. UVVis absorption spectra were recorded on a Purkinje TU-1901 spectrometer. All the Raman measurements were carried out using an Ocean Optics QE65000 Raman spectrometer equipped with a TE-cooled FFT-CCD detector. The excitation wavelength was set as $785 \mathrm{~nm}$ and the input laser power was about $200 \mathrm{~mW}$. The total accumulation time of the SERS measurement was 1 and $5 \mathrm{~s}$ for the fibers and sol, respectively. All the spectra were calibrated with standard silicon wafers as reference to the Raman mode of silicon at $520.7 \mathrm{~cm}^{-1}$.

\subsection{Fabrication of the SERS-active SPME fiber with internal reference}

The synthesis of Au@4-ATP@Ag core-shell NPs is similar to that reported in the literature. ${ }^{35} \mathrm{In}$ a typical run, gold nanoparticles were synthesized through thermal reduction of $\mathrm{HAuCl}_{4}$ using sodium citrate according to a previously published method. ${ }^{36}$ After the reduction of gold chloride, centrifugation was performed to remove the extra sodium citrate ions present in the solution. $0.5 \mathrm{~mL}$ of 4-ATP $(0.01 \mathrm{mM})$ dissolved in ethyl alcohol was added to $10 \mathrm{~mL}$ of as-prepared gold nanoparticles solution under stirring condition. Then the solution was kept overnight at room temperature. Afterwards, hydroquinone $(200 \mu \mathrm{L}$, $10 \mathrm{mM}$ in water) was added to $10 \mathrm{~mL}$ Au@4-ATP solution followed by adding $\mathrm{AgNO}_{3}(200 \mu \mathrm{L}, 10 \mathrm{mM}$ in water) under vigorous stirring. ${ }^{37}$ In order to complete the reaction the solution was kept at room temperature for about $6 \mathrm{~h}$. The precipitates were collected after the centrifugation $(10 \mathrm{~min}$ at 8000 $\mathrm{rpm}$ ) and re-dispersed in $10 \mathrm{~mL}$ of deionized water for later use. Different Au@4-ATP@Ag samples were also prepared by changing the amount of 4-ATP and $\mathrm{AgNO}_{3}$ in order to obtain the optimal value. The Au@Ag NPs were prepared by following the similar procedures without the addition of 4-ATP.

The stainless steel fibers $(3.5 \mathrm{~cm})$ were rinsed with acetone, ethanol and ultrapure water under ultrasonication for $5 \mathrm{~min}$. The ZnO NRs on the fiber were prepared by a hydrothermal reaction according to the report published by Lori $e t ~ a l .{ }^{38}$ The fibers were immersed in a $0.005 \mathrm{M}$ zinc acetate dihydrate solution for $1 \mathrm{~min}$. Then the fibers were dried in argon atmosphere followed by coating step repeated for about three to five times. After that, the fibers were heated to $350{ }^{\circ} \mathrm{C}$ in air for 20 min to yield layers of $\mathrm{ZnO}$ islands. The aligned nanocrystal seeds in aqueous solution at $90{ }^{\circ} \mathrm{C}$ for $4 \mathrm{~h}$ resulted into $\mathrm{ZnO}$ NRs. As an inorganic solid phase microextraction coating, $\mathrm{ZnO}$ NRs own thermally and chemically stability, ${ }^{39}$ and can be used as a good load matrix for the gold and silver materials. ${ }^{40}$ Moreover, ZnO has a good extraction effect for some organic compounds, especially for mercapto compounds. ${ }^{41}$

The Au@4-ATP@Ag core-shell NPs were coupled to the stainless steel fibers coated with ZnO NRs by surface modification using amino groups according to previously published method. ${ }^{40}$ The fibers were immersed into $80 \mathrm{~mL}$ dimethyl sulphoxide (DMSO). Then, $10.8 \mathrm{~g}$ of 3-aminopropyltriethoxysilane (APTES) was added to the solution and the solution was heated for $3 \mathrm{~h}$ at $120{ }^{\circ} \mathrm{C}$. The amino-functionalized ZnO NRs coated fibers were 
taken out from the solution and washed repeatedly with ethyl alcohol. Further, the fibers were immersed into Au@4-ATP@Ag core-shell NPs sol for $10 \mathrm{~h}$. After that, the fibers were rinsed several times with distilled water followed by ethyl alcohol and dried in the air. Two analytes such as malachite green and crystal violet were taken as probe molecules. The analytes were dissolved in water to achieve the desired concentration and also to simulate the aquaculture water.

\section{Results and discussion}

\subsection{Characterization of the Au@4-ATP@Ag NPs}

Fig. 1A shows the TEM image of Au@4-ATP@Ag NPs. It could be observed that the darker nucleus corresponds to the Au NPs and the lighter shell corresponds to the $\mathrm{Ag}$ shell. The average diameter of the nanoparticles was found as $45 \mathrm{~nm}$. The UV-vis spectra of Au@4-ATP@Ag NPs were shown in Fig. 1B. The absorption bands were observed at $400 \mathrm{~nm}$ and also in the wavelength range of 480-510 nm. The spectrum of Au@4ATP@Ag contains a broad band at $400 \mathrm{~nm}$ represents the plasmon band of Ag. A slightly blue shifted Au plasmon band was observed in the $\sim 480-510 \mathrm{~nm}$ wavelength range when compared with the UV-vis spectrum of Au@4-ATP nanoparticles. HRTEM image and elemental mapping of Au@4ATP@Ag NPs can be seen in Fig. S6 and S7. $\uparrow$ These results confirmed the formation of the Ag-shell on the Au@4-ATP. More TEM images of Au@4-ATP@Ag NPs were shown in Fig. S8. $\dagger$

4-ATP was chosen as a probe molecule in this work. The probe molecules were embedded between Au core and Ag shell in the core-shell structure. Thus it makes there is no competition between the analytes and probe molecules for the nanoparticles surface. Moreover, the embedded probe molecules led to achieve an effective feedback to correct the fluctuation of samples and measuring conditions. ${ }^{42}$ The Raman measurements of the prepared samples are shown in Fig. 2. The peaks at 1140, 1391 and $1440 \mathrm{~cm}^{-1}$ were assigned to the b2 modes of the 4-ATP molecule, which has been widely accepted. Their peak intensities have been widely used to estimate the SERS enhancement of some unique substrates. ${ }^{43}$ As shown in Fig. 2, a much higher Raman signal was observed for 4-ATP embedded in the Au@4-ATP@Ag NPs among all the samples. Even same amount of 4-ATP molecules were added, the Raman intensity of 4-ATP molecules adsorbed onto the surface of Au@Ag NPs exhibited a lower signal compared with Au@4-ATP@Ag NPs.
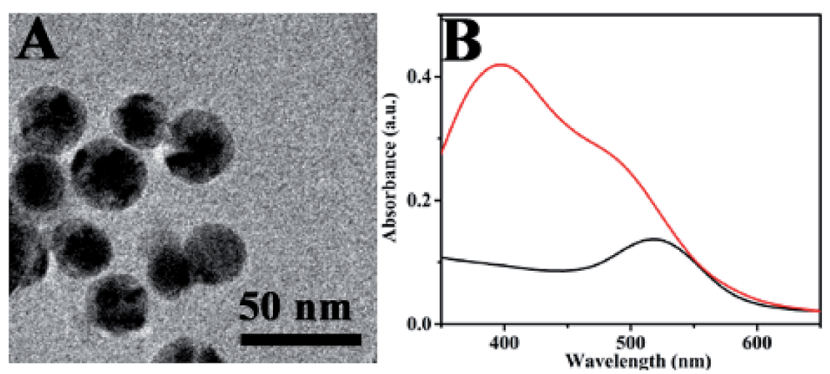

Fig. 1 (A) TEM image of Aua4-ATPaAg NPs. (B) UV-Vis spectra of Au NPs (black) and Au@4-ATP@Ag NPs (red).

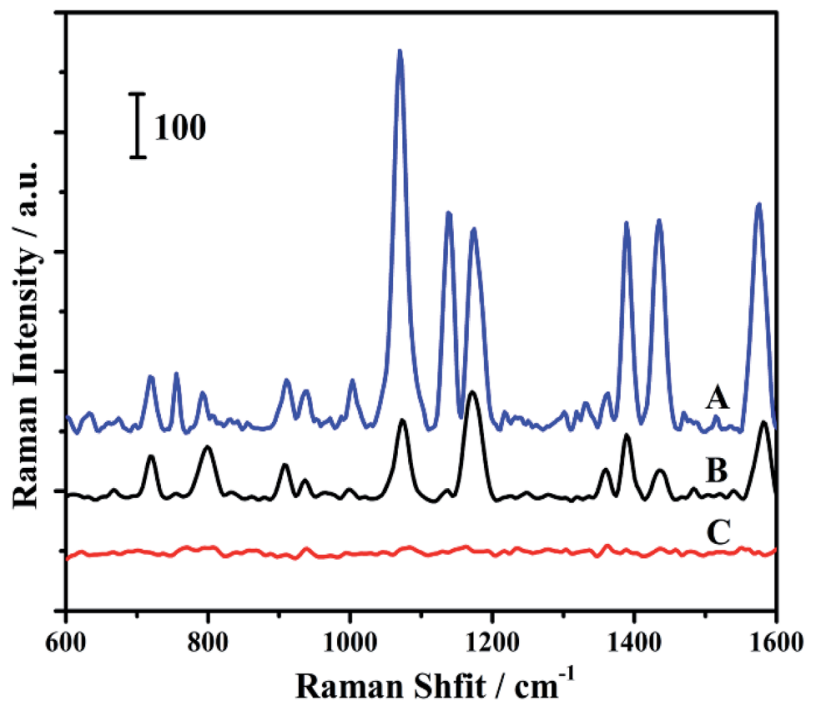

Fig. 2 Raman spectra of Au@4-ATP@Ag nanoparticles (A), Au@Ag nanoparticles with 4-ATP added (B) and Au@Ag nanoparticles (C).

Huang and his co-workers reported that 4-ATP could be selectively and rapidly (in several seconds) oxidized to $p, p^{\prime}$ dimercaptoazobenzene (DMAB) on $\mathrm{Au}$ or $\mathrm{Ag}$ nanoparticles at room temperature during conventional SERS measurements. The obtained b2 mode signals were originated from DMAB rather than 4-ATP. ${ }^{44}$ The b2 mode signals were dropped when compared to the other peaks in the curve B as shown in Fig. 2. It can be explained by the fact that when two 4-ATP molecules are located at a distance far away from each other in solution may lead to reduce the bonding between the molecules. Even if all the molecules adsorbed on the surface nanoparticles, the distance between two molecules was still much longer than that in the shell on account of the larger surface areas of core-shell nanoparticle compared to the core. As a result, the transformation of 4-ATP to DMAB would be more difficult.

The Raman measurements were used to optimize the internal reference molecule amount and silver shell thickness. The results were included in the ESI in Fig. S1 and S2. $\dagger$ The amount of internal reference molecule and also the thickness of the silver shell had an impact on Raman intensity. The peak at $1075 \mathrm{~cm}^{-1}$ has been chosen as internal standard peak, and the other peaks were not considered. With increase in the amount of 4-ATP, the peak height was observed as increased as shown in Fig. S1. $\dagger$ The sol became unstable once the volume increased to $1000 \mu \mathrm{L}$. The amount of 4-ATP was chosen as $500 \mu \mathrm{L}$ in this work.

As shown in Fig. $\mathrm{S} 2, \uparrow$ the Raman signal was observed as increased with the increase in the amount of $\mathrm{AgNO}_{3}$ due to the stronger Raman enhancement ability of $\mathrm{Ag}$ compared to that of $\mathrm{Au}$. When the $\mathrm{AgNO}_{3}$ amount was greater than $2 \mu \mathrm{mol}$, the sol changed into a turbid state. Therefore, $2 \mu \mathrm{mol}$ was selected as the optimized $\mathrm{AgNO}_{3}$ amount.

\subsection{Characterization of the SERS-active SPME fiber}

Fig. 3A shows the SEM images of ZnO NRs grown on the stainless steel fibers. The ZnO NRs were vertically aligned on the 

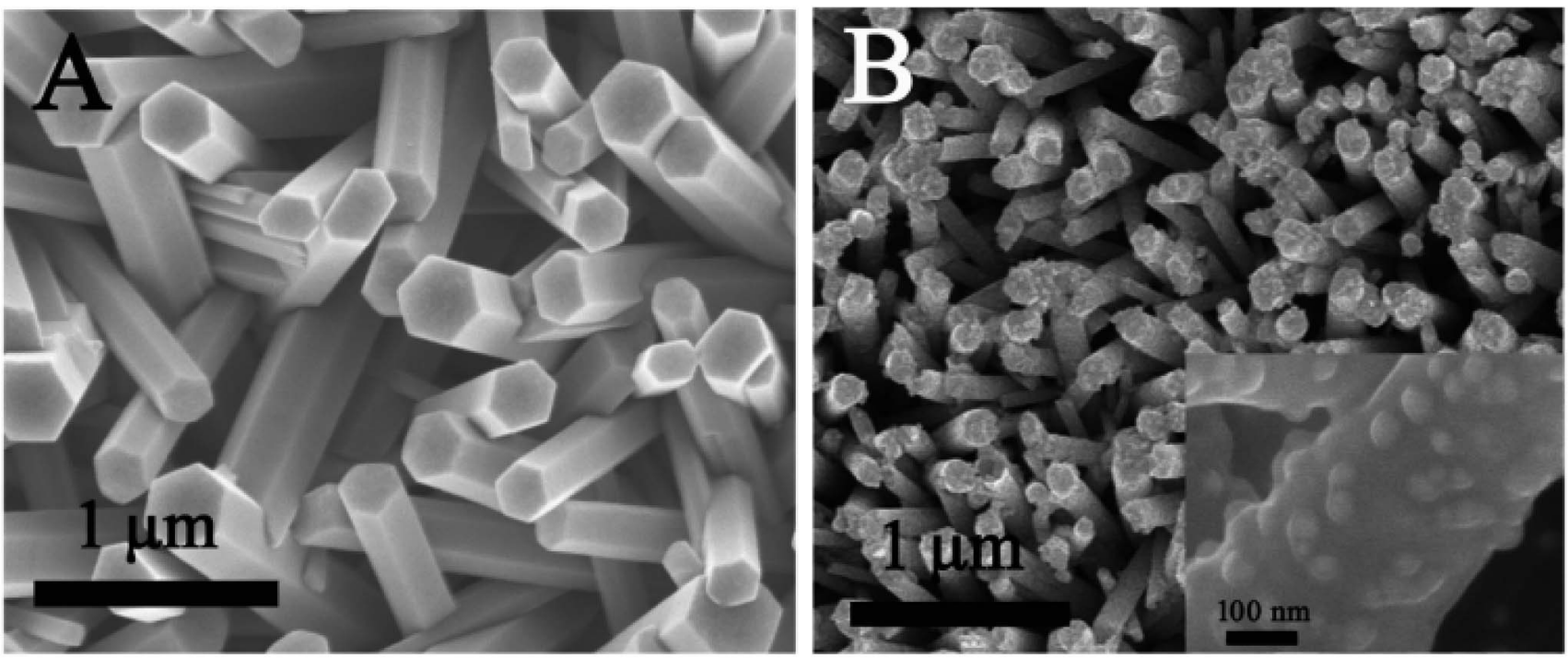

Fig. 3 (A) SEM image of ZnO NRs; (B) SEM image of ZnO NRs with core-shell NPs coupled onto them. Inset: enlarged SEM image.

stainless steel fibers. The rods were in close proximity and formed a uniform 3D structure. Au@4-ATP@Ag NPs were adsorbed on the side and top surfaces of the ZnO NRs by selfassembly, which can be seen clearly in Fig. 3B.

\subsection{Uniformity and stability of the as-prepared SERS-active SPME fiber}

The uniformity of the SERS-active substrates is one of the essential parameters for SERS detection in analytical applications. As shown in Fig. 4A, the SERS spectra of MG at 20 randomly-selected points were recorded to evaluate the uniformity of the as-prepared fiber. The RSD value of the Raman intensity of the MG at $1174 \mathrm{~cm}^{-1}$ was $10.7 \%$. After normalization, the RSD value was reduced to $6.7 \%$, which can be seen in Fig. 4B. The results demonstrated that the normalization effectively reduced the nonuniformity caused by the variation of the adsorbed molecular number, fluctuation of the excitation and scattered field. This can also be proved by the following theoretical calculation. Assuming that a, b, c... are randomly selected spots on the as-prepared fiber, then the Raman intensity could be described by the following equation: ${ }^{45}$

$$
I_{\mathrm{SERS}}=N I_{\mathrm{L}}\left|A\left(v_{\mathrm{L}}\right)\right|^{2}\left|A\left(v_{\mathrm{S}}\right)\right|^{2} \sigma_{\mathrm{ads}}^{\mathrm{R}}
$$

In the formula, $I_{\text {SERS }}$ is the SERS signal, $N$ is the number of adsorption molecules involved in the SERS process, $I_{\mathrm{L}}$ is the excitation intensity, $A\left(v_{\mathrm{L}}\right)$ and $A\left(v_{\mathrm{S}}\right)$ are excitation and scattered field enhancement factors, $\sigma_{\mathrm{ads}}^{\mathrm{R}}$ is the increased Raman cross section of the adsorbed molecule.

$$
N_{\mathrm{a}} \neq N_{\mathrm{b}} \neq N_{\mathrm{c}} \neq N_{\ldots}
$$

The adsorbed molecular number, the fluctuation in the Raman spectrometer, the variation of the excitation and the
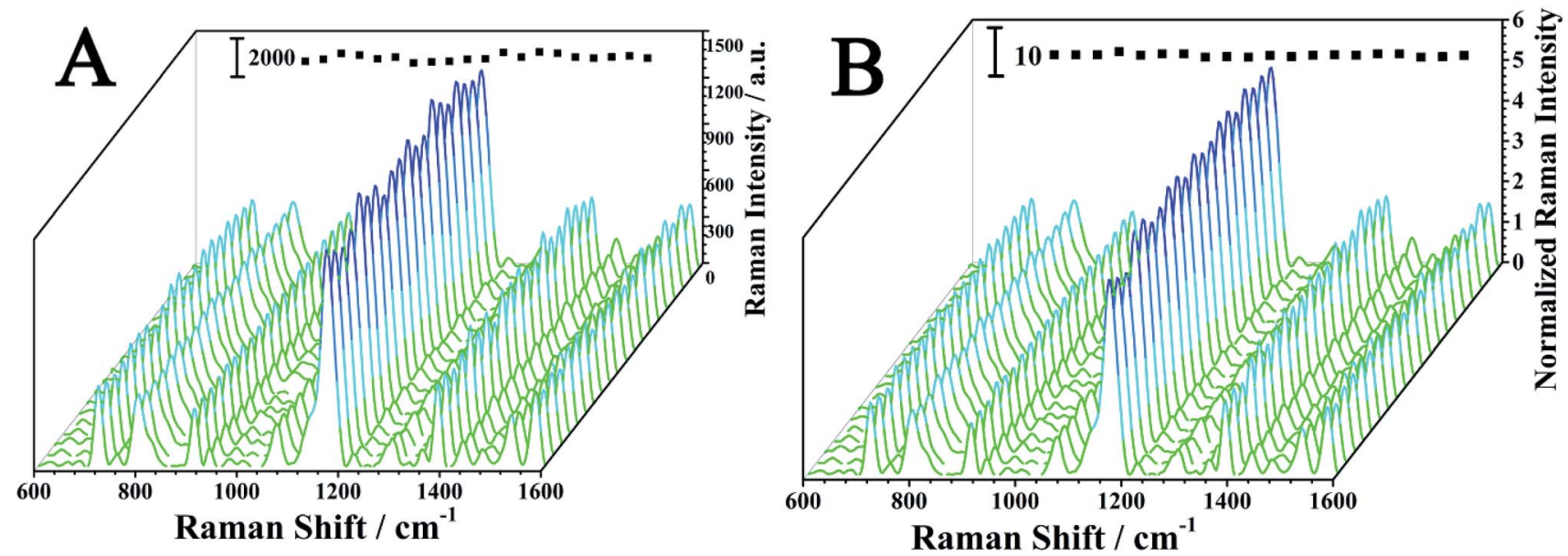

Fig. 4 (A) The SERS spectra of the SERS-active SPME fiber probed with $1.00 \times 10^{-5} \mathrm{M} \mathrm{MG}$ at 20 randomly-selected points. (B) The normalized SERS spectra using the Raman peak of 4-ATP at $1075 \mathrm{~cm}^{-1}$ as reference. The inset images show the changes of the Raman peak of MG at 1174 $\mathrm{cm}^{-1}$. 
scattered field enhancement factor may vary from spot to spot, therefore it led to the SERS intensity fluctuation.

$$
I_{\mathrm{SERS}, \mathrm{a}} \neq I_{\mathrm{SERS}, \mathrm{b}} \neq I_{\mathrm{SERS}, \mathrm{c}} \neq I_{\mathrm{SERS}}, \ldots
$$

Not only the SERS intensity will fluctuate for MG, it may also fluctuate for 4-ATP on the SPME fiber. The RSD of the Raman peak of 4 -ATP at $1075 \mathrm{~cm}^{-1}$ was $15.4 \% . I_{\mathrm{L}}, A\left(v_{\mathrm{L}}\right)$ and $A\left(v_{\mathrm{S}}\right)$ are same for MG and 4-ATP on the spots of the fiber. For a specific molecule, $\sigma^{\mathrm{R}}$ is a constant, hence:

$$
I_{\text {normalized }}=\frac{I_{\mathrm{SERS}, \mathrm{MG}}}{I_{\mathrm{SERS}, 4-\mathrm{ATP}}}=k \frac{N_{\mathrm{MG}}}{N_{4-\mathrm{ATP}}}
$$

Besides, the adsorption process of the molecules in solution could usually be described using the Freundlich isotherm equation: ${ }^{46}$

$$
\begin{gathered}
N=k c^{1 / n} \\
\frac{N_{\mathrm{MG}}}{N_{4-\mathrm{ATP}}}=k_{1} c^{1 / n}
\end{gathered}
$$

The following equation can be obtained from eqn (4) and (6):

$$
I_{\text {normalized }}=k k_{1} c^{1 / n}
$$

where $c$ is the concentration of the MG, $k, k_{1}$ and $n$ are the constants for a certain system at a certain temperature.

This suggests that, the Raman intensity ratio of MG to 4-ATP for different spots may be equal in a certain solution.

The SERS spectra of MG were recorded for 10 min under continuous laser radiation with laser power at $200 \mathrm{~mW}$ to evaluate the stability of the fiber, as given in Fig. 5. It could be seen that the Raman signals of the SERS-active SPME fiber under continuous laser radiation for $10 \mathrm{~min}$ had no obvious change. The RSD value of the intensity of the Raman peak of MG at $1174 \mathrm{~cm}^{-1}$ was $6.8 \%$. The SERS spectra of MG after the fibers being stored in air for 2 months were also recorded, which

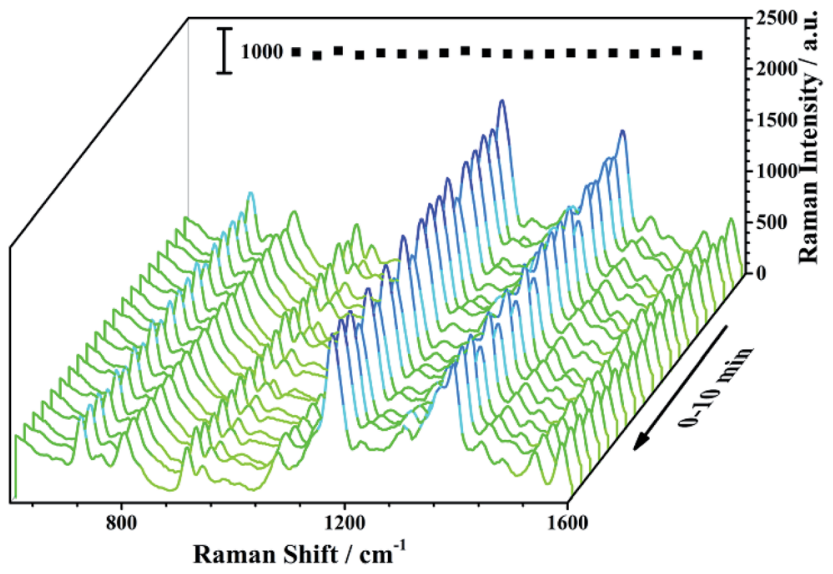

Fig. 5 SERS spectra of the SERS-active SPME fiber probed with $1.00 \times$ $10^{-5}$ M MG under continuous laser radiation. The inset image shows the changes of the Raman peak of MG at $1174 \mathrm{~cm}^{-1}$. showed the Raman intensity of MG at $1174 \mathrm{~cm}^{-1}$ only dropped $2.40 \%$, as demonstrated in Fig. S5. $\dagger$ It was suggested that the SERS-active SPME fiber exhibits good stability.

\subsection{Optimization of the extraction time}

To investigate the extraction ability of the fibers, the fibers were immersed into $1 \times 10^{-8} \mathrm{M}$ MG solution and the spectra were recorded at regular intervals. The kinetics of MG adsorption can be seen in Fig. S3. $\dagger$ The extraction efficiency increased with increase in extraction time before it reached the adsorption equilibrium. For the solution with $1 \times 10^{-8} \mathrm{M}$ initial MG concentration, the saturated adsorption time was about $180 \mathrm{~min}$. After $120 \mathrm{~min}$, it was observed that the adsorption process tends to be saturated. When the concentration of the sample increased, the time required to reach the adsorption equilibrium state would also increase. Hence, the extraction time was taken as $120 \mathrm{~min}$ in the following experiments.

\subsection{Qualitative SERS detection of MG}

MG is one of the most widely used fungicides in the fish farming and has been banned for its potential animal mutagenicity and teratogenicity. ${ }^{47-49}$ Therefore, it is important to monitor the concentration of MG. The SERS spectrum of MG on the fiber, the Raman spectrum of the MG solution and the SERS spectrum of the blank fiber were shown in Fig. 6. The SERS spectrum of MG was comparable with the Raman spectrum of MG solution. It suggested that MG had been extracted onto the fiber surface. The Raman spectrum of MG showed characteristic Raman bands at 800, 916, 1174, 1219, 1292, 1396, $1442 \mathrm{~cm}^{-1}$. The peak located at $1442 \mathrm{~cm}^{-1}$ was assigned to in-plane $\mathrm{C}-\mathrm{C}$ stretching vibration of the ring. The broadened peaks appeared at $1396 \mathrm{~cm}^{-1}$ were attributed to the synergy of in-plane $\mathrm{C}^{+}-\mathrm{C}$ stretching vibration and $N$-phenyl stretching. The peak located at $916 \mathrm{~cm}^{-1}$ corresponded to the skeleton vibration of radial aromatic ring. The peaks at 800 and $1174 \mathrm{~cm}^{-1}$ were assigned as

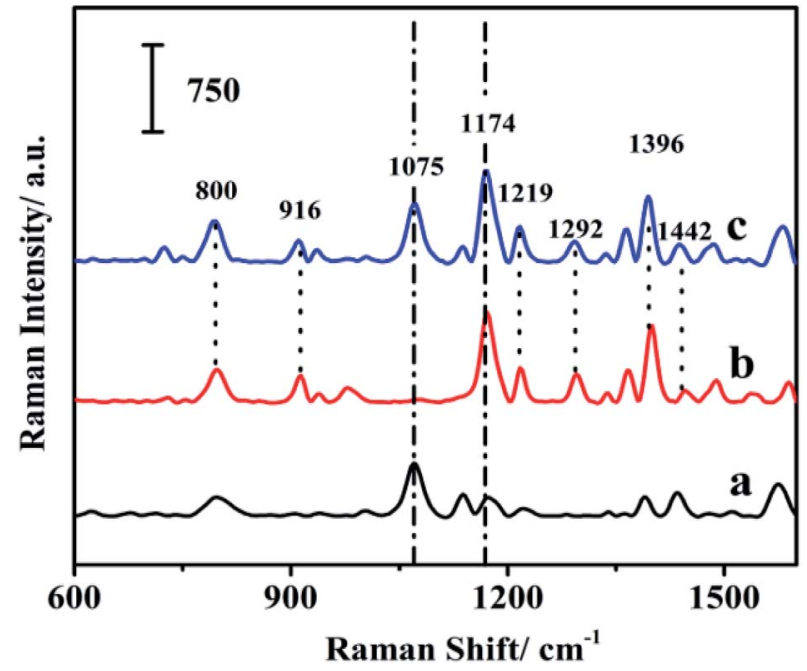

Fig. 6 Raman signals of the fiber with internal reference (a); MG solution (b); and MG extracted onto the fiber (c). 
the bending vibration of radial aromatic ring in the plane of $\mathrm{C}-\mathrm{H}$ bond. ${ }^{50}$ All these peaks were also identified in the SERS spectrum of MG on the fiber, which indicated that MG had been successfully extracted onto the as-prepared SERS-active SPME fiber.

The characteristic Raman band of 4-ATP at $1075 \mathrm{~cm}^{-1}$ was chosen as the internal standard Raman peak. The characteristic Raman band of MG at $1174 \mathrm{~cm}^{-1}$ was chosen as the quantitative peak to quantitatively detect MG.

And the enhancement factor (EF) was estimated according to the following formula:

$$
\mathrm{EF}=\frac{I_{\text {SERS }}}{I_{\text {Raman }}} \times \frac{N_{\text {Raman }}}{N_{\text {SERS }}}
$$

where $I_{\text {SERS }}$ represents the SERS intensity of the characteristic peak for the analytes adsorbed on fiber surface, $I_{\text {Raman }}$ represents normal Raman intensity of the same peak from the analytes solution. $N_{\text {Raman }}$ and $N_{\text {SERS }}$ are the number of the analytes in the illumination volume in the solution and on the fiber, respectively. $I_{\text {SERS }}$ and $I_{\text {Raman }}$ are measured from the peak at $1174 \mathrm{~cm}^{-1}$ for MG. EF of MG was calculated as $1.11 \times 10^{5}$.

\subsection{Quantitative SERS detection of MG}

To investigate the quantitative ability of the fibers, the fibers were immersed into the MG solution with concentration range of $1 \times 10^{-7} \mathrm{M}$ to $5 \times 10^{-7} \mathrm{M}$. The laser detection sites were randomly selected on the fibers, which may lead to fluctuation in Raman intensity. As shown in Fig. 7A, the SERS intensity of the $\mathrm{MG}$ at $1174 \mathrm{~cm}^{-1}$ increased with an increase in $\mathrm{MG}$ concentration. The log-log plot of the Raman intensity of MG at $1174 \mathrm{~cm}^{-1}$ versus its concentration showed a linear relationship as shown in Fig. 7B. The linear equation was $y=10.775+1.136 x$ ( $x$ is the logarithm of the MG concentration, $y$ is the logarithm of the SERS intensity of the Raman peak at $1174 \mathrm{~cm}^{-1}$ ), and the correlation coefficient $\left(R^{2}\right)$ was found as 0.962 . When the peaks were normalized according to the internal reference peak of 4ATP at $1075 \mathrm{~cm}^{-1}$, the normalized Raman intensity versus the MG concentration showed much better linearity. The correlation coefficient $R^{2}$ was observed as improved to 0.984 from 0.962 . The linear equation was $y=5.691+0.772 x(x$ is the logarithm of the MG concentration, $y$ is logarithm of ratio of the SERS intensity of the Raman peak at 1174 and $1075 \mathrm{~cm}^{-1}$ ). This indicates the importance of using the internal reference method in quantitative measurements. The above results suggest that the internal reference signal can effectively correct the signal fluctuation as a result of the different adsorbed molecular number, the fluctuation in the Raman spectrometer, the variation of the excitation and the scattered field enhancement factor.

The relationship between the SERS intensity and the adsorbed molecule number can be obtained from eqn (1) and (5):

$$
\begin{gathered}
I_{\text {SERS }}=a N=a k c^{1 / n}=K c^{1 / n} \\
\log I_{\text {SERS }}=\frac{1}{n} \log c+\log K
\end{gathered}
$$

where $n$ and $K$ are constants and not related to MG concentration.

From eqn (9), we can know that the good linear relationship of the log-log plot for SERS intensity to analyte concentration is reasonable.

\subsection{SERS quantification of MG and $\mathrm{CV}$ in aquaculture water}

Crystal violet (CV) is another triphenylmethane dye which can cause carcinogenicity in humans. ${ }^{51,52}$ The detection of $\mathrm{CV}$ in aquaculture water was carried out in order to check the ability of the SERS-active SPME fiber for the practical applications. As can be seen from Fig. $\mathrm{S} 4, \dagger$ the structures of MG and CV are very
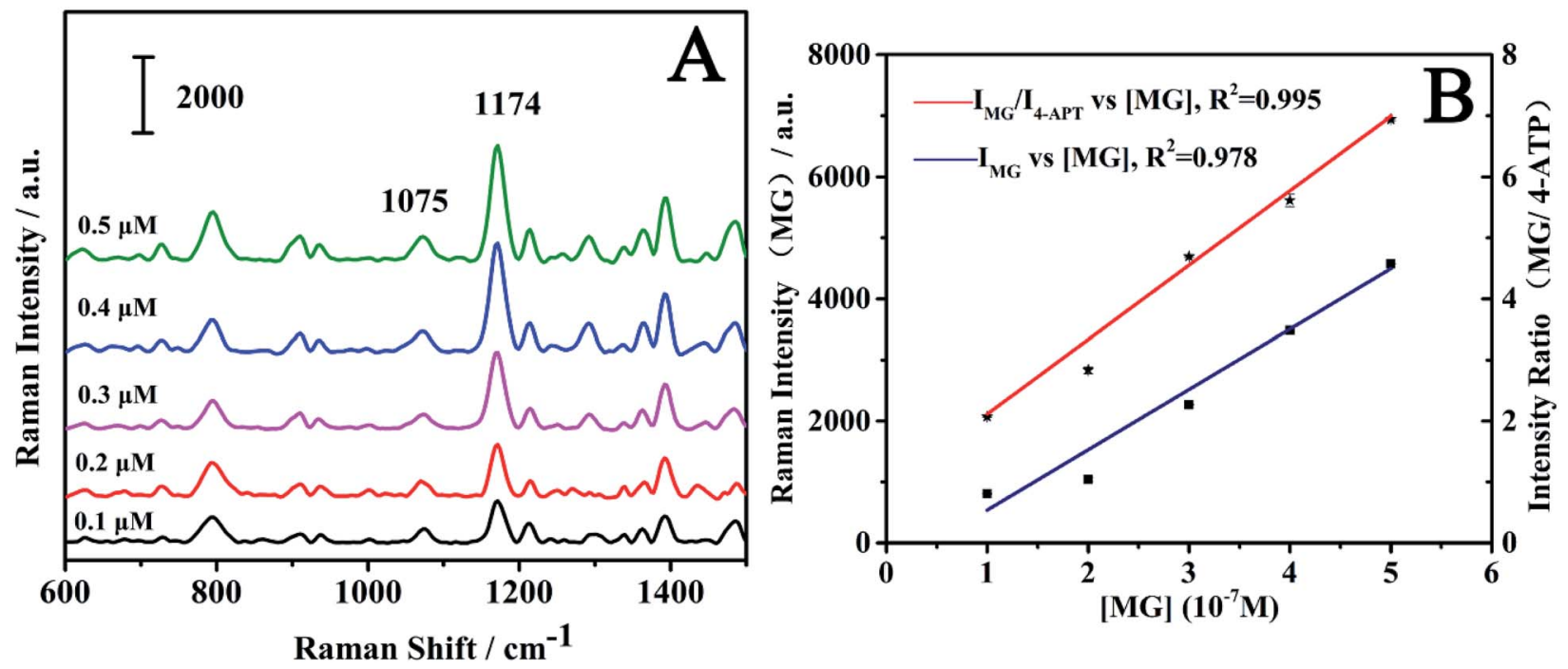

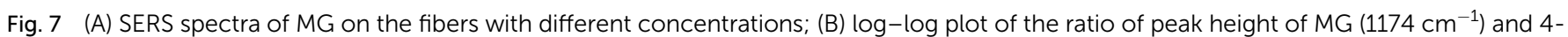
ATP $\left(1075 \mathrm{~cm}^{-1}\right)$ vs. MG concentration (red); log-log plot of peak height of MG $\left(1174 \mathrm{~cm}^{-1}\right)$ vs. MG concentration (blue). The data points were obtained from three parallel measurements at each concentration. 

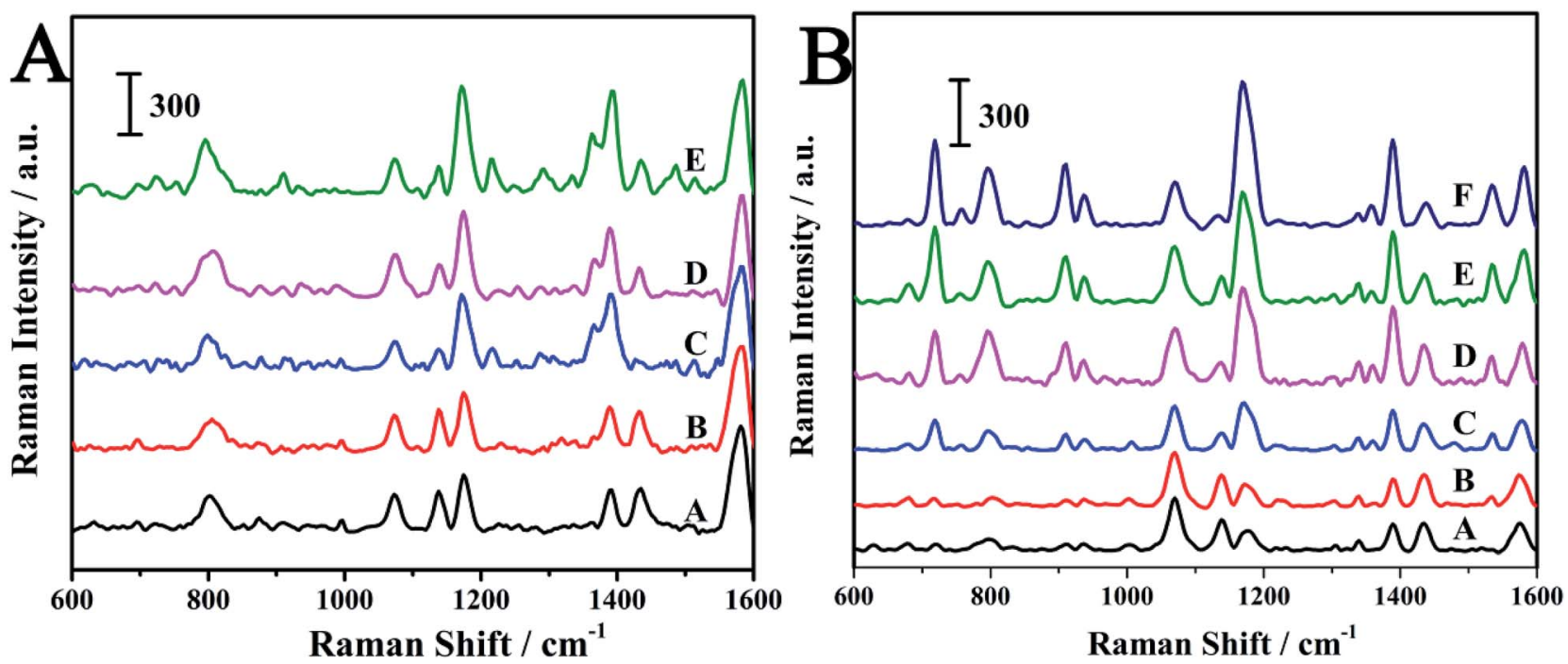

Fig. 8 (A) SERS spectra of MG at different concentrations in aquaculture water: (A) $0.5 \mu \mathrm{M}$; (B) $1 \mu \mathrm{M}$; (C) $2.5 \mu \mathrm{M}$; (D) $5 \mu \mathrm{M}$; (E) $50 \mu \mathrm{M}$. (B) SERS spectra of CV at different concentrations in aquaculture water: (A) $0.05 \mu \mathrm{M}$; (B) $0.1 \mu \mathrm{M}$; (C) $0.5 \mu \mathrm{M}$; (D) $1 \mu \mathrm{M}$; (E) $2.5 \mu \mathrm{M}$; (F) $5 \mu \mathrm{M}$.
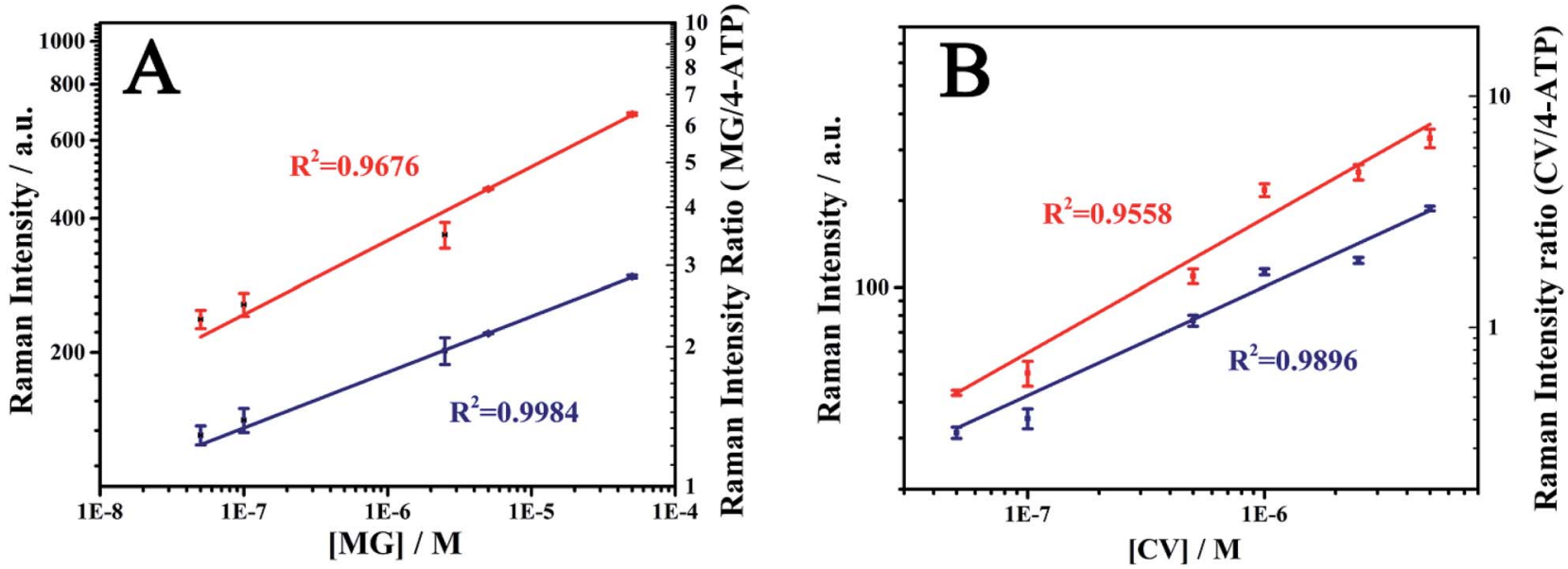

Fig. $9 \log$-log plot for MG (A) and CV (B). The red line showed the Raman intensity vs. concentration; the blue line showed the Raman intensity ratio vs. concentration. The data points were obtained from three parallel measurements at each concentration.

similar. The characteristic peak of CV was also observed at 1174 $\mathrm{cm}^{-1}$. The MG and $\mathrm{CV}$ was dissolved in aquaculture water to prepare various concentrations and then detected by the SERSactive SMPE fibers. The SERS spectra of MG and CV at different concentrations were shown in Fig. 8. The SERS intensity of the analytes at $1174 \mathrm{~cm}^{-1}$ was increased with increase in the concentration. The log-log plot of the Raman intensity of at $1174 \mathrm{~cm}^{-1}$ versus its concentration and the log-log plot of the Raman intensity ratio versus analytes concentration were shown in Fig. 9. As is seen in Fig. 9, the normalized Raman intensity showed better linear relationship with the concentration. And from the results given in Table. $\mathrm{S} 1, \uparrow$ it can be comprehensible that the fiber had better quantitative analysis ability compared to other SERS sensors. In contrast to other methods in Table $\mathrm{S} 2, \uparrow$ this technique showed a comparable detection limit. The above results suggest the advantage of the internal reference method and its feasibility in practical application.

\section{Conclusions}

In summary, Au@4-ATP@Ag NPs coated ZnO NRs on a stainless steel fiber was prepared as a SERS-active SPME fiber to quantitatively measure the SERS signals of analytes. The prepared SERSactive SPME fiber exhibited good uniformity, stability and extraction ability which indicates its potential for routine analysis. The log-log plot of SERS intensity to MG concentration showed a good linear relationship and the $R^{2}$ before and after the normalization was 0.962 and 0.984 , respectively. The results indicated that the internal reference signal can effectively correct the signal fluctuation, which provides a reliable quantitative SERS 
analysis. The quantitative detection of $\mathrm{CV}$ in aquaculture water was also demonstrated for the practical applications. In the concentration range of $5 \times 10^{-8} \mathrm{M}$ to $5 \times 10^{-6} \mathrm{M}$, the $R^{2}$ after normalization was 0.990 with the detection limit of $1.9 \times 10^{-9} \mathrm{M}$ $(\mathrm{S} / \mathrm{N}=3)$. From the above results, it can be concluded that the SERS-active SPME fiber reported in this work provided a new method for on-site quantitative SERS measurements.

\section{Acknowledgements}

We are grateful for the financial support from the National Basic Research Program of China (973 Program 2013CB934301), the National Natural Science Foundation of China (NSFC21377068, 21575077), the Natural Science Foundation of Shandong Province of China (no. ZR2014BM033) and the Fundamental Research Funds of Shandong University (2016JC030).

\section{Notes and references}

1 C. Baronti, R. Curini, G. D'Ascenzo, A. Di Corcia, A. Gentili and R. Samperi, Environ. Sci. Technol., 2000, 34, 5059-5066.

2 L. H. Santos, A. N. Araújo, A. Fachini, A. Pena, C. DelerueMatos and M. Montenegro, J. Hazard. Mater., 2010, 175, 45-95.

3 C. Tixier, H. P. Singer, S. Oellers and S. R. Müller, Environ. Sci. Technol., 2003, 37, 1061-1068.

4 M. d. F. Alpendurada, J. Chromatogr. A, 2000, 889, 3-14.

5 C. L. Arthur and J. Pawliszyn, Anal. Chem., 1990, 62, 21452148.

6 H. Lord and J. Pawliszyn, J. Chromatogr. A, 2000, 885, 153193.

7 X. Yu, H. Yuan, T. Górecki and J. Pawliszyn, Anal. Chem., 1999, 71, 2998-3002.

8 O. Pinho, I. Ferreira and M. Ferreira, Anal. Chem., 2002, 74, 5199-5204.

9 S. Li and S. G. Weber, Anal. Chem., 1997, 69, 1217-1222.

10 W. M. Mullett, K. Levsen, J. Borlak, J. Wu and J. Pawliszyn, Anal. Chem., 2002, 74, 1695-1701.

11 D. L. Heglund and D. C. Tilotta, Environ. Sci. Technol., 1996, 30, 1212-1219.

12 S. A. Merschman, S. H. Lubbad and D. C. Tilotta, J. Chromatogr. A, 1998, 829, 377-384.

13 D. A. Long, Raman spectroscopy, New York, 1977, pp. 1-12.

14 B. L. Wittkamp and D. C. Tilotta, Anal. Chem., 1995, 67, 600605.

15 D. Li, L. Qu, W. Zhai, J. Xue, J. S. Fossey and Y. Long, Environ. Sci. Technol., 2011, 45, 4046-4052.

16 Y.-T. Li, L.-L. Qu, D.-W. Li, Q.-X. Song, F. Fathi and Y.-T. Long, Biosens. Bioelectron., 2013, 43, 94-100.

17 D.-W. Li, W.-L. Zhai, Y.-T. Li and Y.-T. Long, Microchim. Acta, 2014, 181, 23-43.

18 M. Odziemkowski, J. A. Koziel, D. E. Irish and J. Pawliszyn, Anal. Chem., 2001, 73, 3131-3139.

19 C. J. Constantino, T. Lemma, P. A. Antunes and R. Aroca, Anal. Chem., 2001, 73, 3674-3678.

20 S. Nie and S. R. Emory, Science, 1997, 275, 1102-1106.
21 J. Tsang, J. Kirtley and J. Bradley, Phys. Rev. Lett., 1979, 43, 772.

22 S. S. Dasary, A. K. Singh, D. Senapati, H. Yu and P. C. Ray, J. Am. Chem. Soc., 2009, 131, 13806-13812.

23 L. Guerrini, J. V. Garcia-Ramos, C. Domingo and S. SanchezCortes, Anal. Chem., 2009, 81, 1418-1425.

24 F. Inscore, C. Shende, A. Sengupta, H. Huang and S. Farquharson, Appl. Spectrosc., 2011, 65, 1004-1008.

25 R. M. Jarvis and R. Goodacre, Chem. Soc. Rev., 2008, 37, 931936.

26 D. Li, D.-W. Li, J. S. Fossey and Y.-T. Long, Anal. Chem., 2010, 82, 9299-9305.

27 M. Lin, L. He, J. Awika, L. Yang, D. R. Ledoux, H. a. Li and A. Mustapha, J. Food Sci., 2008, 73, T129-T134.

28 R. J. Stokes, E. McBride, C. G. Wilson, J. M. Girkin, W. E. Smith and D. Graham, Appl. Spectrosc., 2008, 62, 371-376.

29 B. Li, Y. E. Shi, J. Cui, Z. Liu, X. Zhang and J. Zhan, Anal. Chim. Acta, 2016, 923, 66-73.

30 C. Liu, X. Zhang, L. Li, J. Cui, Y. E. Shi, L. Wang and J. Zhan, Analyst, 2015, 140, 4668-4675.

31 S. Zhu, X. Zhang, J. Cui, Y. E. Shi, X. Jiang, Z. Liu and J. Zhan, Analyst, 2015, 140, 2815-2822.

32 S. M. Stranahan and K. A. Willets, Nano Lett., 2010, 10, 37773784 .

33 H. Xu, E. J. Bjerneld, M. Käll and L. Börjesson, Phys. Rev. Lett., 1999, 83, 4357.

34 S. E. Bell and N. M. Sirimuthu, Chem. Soc. Rev., 2008, 37, 1012-1024.

35 Y. Zhou, R. Ding, P. Joshi and P. Zhang, Anal. Chim. Acta, 2015, 874, 49-53.

36 G. Frens, Nature, 1973, 241, 20-22.

37 Z. Chen, D. Yu, Y. Huang, Z. Zhang, T. Liu and J. Zhan, Sci. Rep., 2014, 4, 6709.

38 L. E. Greene, M. Law, D. H. Tan, M. Montano, J. Goldberger, G. Somorjai and P. Yang, Nano Lett., 2005, 5, 1231-1236.

39 R. Alizadeh, N. M. Najafi and S. Kharrazi, Anal. Chim. Acta, 2011, 689, 117-121.

40 P. Chen, L. Gu, X. Xue, Y. Song, L. Zhu and X. Cao, Mater. Chem. Phys., 2010, 122, 41-48.

41 D. J. Djozan and L. Abdollahi, Chromatographia, 2003, 57, 799-804.

42 W. Shen, X. Lin, C. Jiang, C. Li, H. Lin, J. Huang, S. Wang, G. Liu, X. Yan, Q. Zhong and B. Ren, Angew. Chem., 2015, 54, 7308-7312.

43 M. Osawa, N. Matsuda, K. Yoshii and I. Uchida, J. Phys. Chem., 1994, 98, 12702-12707.

44 Y.-F. Huang, H.-P. Zhu, G.-K. Liu, D.-Y. Wu, B. Ren and Z.-Q. Tian, J. Am. Chem. Soc., 2010, 132, 9244-9246.

45 K. Kneipp, H. Kneipp, I. Itzkan, R. R. Dasari and M. S. Feld, Chem. Rev., 1999, 99, 2957-2976.

46 J. Collip, Can. Med. Assoc. J., 1925, 15, 448.

47 A. Afkhami, R. Moosavi and T. Madrakian, Talanta, 2010, 82, 785-789.

48 M. Oplatowska, R. F. Donnelly, R. J. Majithiya, D. Glenn Kennedy and C. T. Elliott, Food Chem. Toxicol., 2011, 49, 1870-1876. 
49 S. Srivastava, R. Sinha and D. Roy, Aquat. Toxicol., 2004, 66, 319-329.

50 E. Liang, X. Ye and W. Kiefer, J. Phys. Chem. A, 1997, 101, 7330-7335.
51 C. Diamante, W. F. Bergfeld, D. V. Belsito, C. D. Klaassen, J. G. Marks, R. C. Shank, T. J. Slaga, P. W. Snyder and F. A. Andersen, Int. J. Toxicol., 2009, 28, 193S-204S. 52 I. Šafařlk and M. Šafařiková, Water Res., 2002, 36, 196-200. 